\title{
Relation between HLA DRB1 alleles and corticosteroid resistance in giant cell arteritis
}

\author{
O Rauzy, M Fort, F Nourhashemi, L Alric, H Juchet, M Ecoiffier, M Abbal, D Adoue
}

\begin{abstract}
Objective-To evaluate the clinical usefulness of genomic HLA typing during the first two years of established giant cell arteritis (GCA).

Methods-HLA typing was performed by PCR-SSO in 41 selected white patients with GCA confirmed by biopsy. Patient data were compared with those of a control group of 384 bone marrow donors (relative risk, $p$ value and $\chi^{2}$ test for each allele). Clinical features at onset and response to treatment over a two year period were evaluated in relation to the genetic pattern. Results-DRB1*04 was significantly increased in the GCA group (frequency of $\mathbf{4 8 . 7 8 \%}$ compared with $19.79 \%$ in controls, $p<0.001)$. The distribution of the DRB1*04 subtypes in the GCA group was similar to that in controls. No clinical or biological differences were found in association with HLA at the time of diagnosis. Over the two year follow up, nine patients presented resistance to corticosteroid treatment and eight of these $(88.88 \%)$ had DRB1*04 (p < 0.001)

Conclusions-GCA seems to be associated with HLA DRB1*04 (regardless of the subtype) and this association appears to be accompanied by corticosteroid resistance, suggesting that genomic typing may be useful to identify patients eligible for early alternative treatment to corticosteroid drugs.

(Ann Rheum Dis 1998;57:380-382)
\end{abstract}

Department of Internal Medicine, CHU Toulouse,

Toulouse, France

O Rauzy

F Nourhashemi

L Alric

H Juchet

M Ecoiffier

D Adoue

Immunology

Laboratory, CHU

Toulouse, Toulouse,

France

M Abbal

M Fort

Correspondence to: Professor D Adoue, Centre de Médecine Interne, $\mathrm{CHU}$ Toulouse, Pavillon Junod, 170 Avenue de Casselardit, 31300 Toulouse, France.

Accepted for publication 7 May 1998 molecular mechanisms underlying the development of GCA are unknown but genetic factors are probably involved in its pathogenesis. ${ }^{12}$ The results of several immunogenetic studies have supported an association of HLA DR4 (DR4 antigens, $\mathrm{DRB} 1^{\star} 04$ sequences) with GCA. ${ }^{3}$ Among GCA patients, a few may have corticosteroid resistant disease. ${ }^{4}$ It would be very useful for clinicians caring for patients with GCA to be able to identify those who are destined to develop resistant disease in order to plan a rational alternative approach to treatment. Whereas some severe chronic systemic diseases, such as rheumatoid arthritis, have been reported to be associated with HLA DR in several cross sectional studies of patients with disease of long duration, ${ }^{5}$ studies of the prognostic value of HLA DR typing in GCA have not given the same results: prospective studies have afforded little information concerning the influence of genetic markers on the subsequent course of established GCA. ${ }^{126}$ Can HLA typing identify patients eligible for early aggressive treatment? The question arises whether more precise DNA typing technology could provide better information.

\section{Methods}

PATIENTS

We investigated 41 patients who were taking part in a continuing prospective study of GCA by the Auto-Immunity Group of the University Hospital of Toulouse, France. The inclusion criterion was confirmed GCA. In all patients, biopsy specimens showed histological temporal artery abnormalities of vasculitis with mononuclear cell infiltration or granulomatous inflammation with or without multinucleated giant cells. This criterion defined the patient cohort. ${ }^{7}$ The patients were eight men and 33 women, all white, with a mean age of 77 years (range 61 to 97 ) at disease onset. All patients received corticosteroid treatment initially for three to four weeks (over the severity of disease an initial amount of prednisone 0.6 to $1 \mathrm{mg} / \mathrm{kg} /$ day in simple divided daily dose) The starting dose was continued until all reversible clinical symptoms have gone and laboratory test have reverted to normal. After that the dose were gradually reduced by a maximum of $5 \mathrm{mg}$ each week or every two weeks. The reduction programme is gauged by the clinical symptoms and blood test (erythrocyte sedimentaion rate (ESR), C reactive protein (CRP), fibrinaemia, interleukin 6 (IL6)). At some point in the reduction programme, when clinical symptoms worsened or biological anomalies rise above normal once again, the further reduction was temporalily deferred and the dose was increased to the earlier level. ${ }^{4}$

\section{DNA ANALYSIS}

HLA typing was performed by PCR-SSO using the set of probes and primers recommended by the XII ${ }^{\text {th }}$ International Workshop on Histocompatibility. ${ }^{8}$ The DRB1 generic specificities (DRB1 $\left.{ }^{\star} 1-16\right)$ were carried out according to the Nomenclature for Factors of the HLA System, 1996. ${ }^{9}$ This study was performed in GCA patients and in a control 
Table 1 Therapeutic status in GCA patients defined by HLA DRB1*04 groups

\begin{tabular}{llll}
\hline & $\begin{array}{l}\text { 20 GCA patients } \\
\text { with DRB1*04 }\end{array}$ & $\begin{array}{l}\text { 21 GCA patients } \\
\text { without DRB1*04 }\end{array}$ & \\
\hline Corticosteroid $>20 \mathrm{mg} /$ day $(\mathrm{n}=9)$ & 8 & 1 & $\mathrm{p}<0.001$ \\
Corticosteroid $<20 \mathrm{mg} /$ day $(\mathrm{n}=32)$ & 12 & 20 &
\end{tabular}

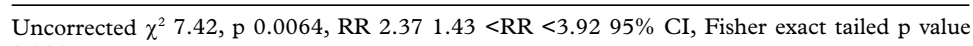
0.008 .
THERAPEUTIC STATUS

Table 1 shows therapeutic status after two years of treatment in GCA patients with or without HLA DRB $1^{\star} 04$. Of the 41 patients, nine were receiving more than $20 \mathrm{mg} /$ day of prednisone. This high dose was required because of clinical exacerbation in one patient (temporal headache), abnormal laboratory findings (ESR, CRP, fibrinaemia, IL6 above 15\% normal without any other explanations) in six, and both (headache, ESR, CRP, fibrinaemia) in two patients. Of the other 32 patients, five were receiving $15 \mathrm{mg} /$ day of prednisone and this dose was being tapered without untoward clinical and biological effects, 19 were receiving $10 \mathrm{mg} /$ day of prednisone and treatment was being discontinued, while the other 17 patients had stopped taking corticosteroids. The HLA $\mathrm{DRB}{ }^{\star} 04$ antigens (whatever the subtype) were associated with corticosteroid requirement above $20 \mathrm{mg} /$ day of prednisone. (88.88\% versus $37.5 \%$; uncorrected $\chi^{2} 7.42, \mathrm{p}=0.0064$, RR $2.371 .43<\mathrm{RR}<3.92,95 \mathrm{CI} \%$, Fisher exact tailed $\mathrm{p}$ value 0.008$)$.

\section{Discussion}

Several studies have found GCA to be associated with HLA DRB $1^{\star} 04 .^{1-3}$ Like ourselves, Weyand ${ }^{3}$ determined HLA DRB1 alleles by oligotyping in 42 patients with GCA confirmed by biopsy and HLA DR4 was found in $60 \%$ of GCA patients and in $24 \%$ of normal controls (our results were respectively $49 \%$ and $20 \%$ ). Some authors found the increase in DR4 frequency was accounted for by DRB1*0401-406 subtypes and when investigators then determined the HLA DRB ${ }^{\star} 1$ alleles in GCA patients lacking DR4, they found an excess of HLA DRB $1{ }^{\star} 3$ and HLA DRB $1{ }^{\star} 08 .{ }^{35}$ Our results are different but we note that the inclusion criteria were not the same in all studies (all our patients had verified GCA with compatible histological features ${ }^{7}$ and this was not an obligatory criterion in most studies).

We found no useful clinical correlation between HLA markers and clinical or biological presentation of GCA at the time of diagnosis. $\mathrm{Cid}^{11}$ found that HLA DRB ${ }^{\star} 04$ association was only seen in GCA patients with polymyalgia rheumatica symptoms. Our study did not support this interpretation: in our cohort of patients with biopsy confirmed GCA, an HLA DRB $1^{\star} 04$ association was also found in patients who lacked polymyalgia rheumatica symptoms. Because of the relative frequency of HLA DRB $1{ }^{\star} 04$ in the normal population, this genetic marker cannot be used as a diagnostic aid. ${ }^{135}$ However, its increased frequency in GCA is a possible argument in favour of an immunogenetic mechanism, ${ }^{312}$ which intervenes in the onset of the disease by promoting a particular presentation of arterial antigens modified by the action of ultraviolet light or by some other environmental factor. ${ }^{2}{ }^{13}$

Corticotherapy is an important element in judging the evolution of GCA; its spectacular effect can be a diagnostic test. In the medical literature, exacerbations of the treated disease

vary greatly in frequency according to the the disease at the time of diagnosis. Similarly, these markers were not correlated with the initial severity of the disease. 
series and often seem related to excessively economical use of corticosteroids. ${ }^{4}$ Exacerbations are more frequent during the first year of treatment and are characterised by renewed systemic manifestations together with the reappearance of the biological inflammatory syndrome. ${ }^{40}$ Late exacerbations also occur when attempts are made to decrease corticosteroid dose, as whatever the treatment regimen, the rule is to aim for the minimal effective dose that will keep patients free from symptoms and with a normal ESR. ${ }^{4}$ The outcome and duration of corticosteroïd treatment in GCA are still debated. Current estimates of outcome are derived from reports of treated patients. Such studies have considerable variations in design and content. The ideal study would be prospective and include a substantial number of patients meeting accepted diagnostic criteria. Treatment regimens would be applied prospectively and response would be judged by predetermined objective criteria defining remission, disease activity, and exacerbation, with long term follow up (that is, at least two years). Such studies are very difficult to carry out. ${ }^{10}$ Although there is great individual variation, the required duration of treatment for most patients seems to be about two years. ${ }^{10}$ It may be accepted that patients who require prolonged corticosteroid treatment with more than $20 \mathrm{mg}$ of prednisone per day will suffer a variety of adverse effects. ${ }^{14}$ These patients are a minority of all GCA patients and may be considered to have corticosteroid resistant disease. ${ }^{10}$ The number of such corticosteroid resistant GCA patients in our study (9 of 41 patients, $22 \%$ even though some of this called "corticoresistant GCA" can stop taking corticosteroids after 3-6 years) is because of a recruitment bias: our group was treated principally for this difficult systemic disease and non-complicated GCA is not usually referred to us. Most clinicians treating GCA patients abstain from giving corticosteroid exclusively based on laboratory findings but with the severity of our patients disease, we prefer to dicuss treatment according to both clinical and biological evaluation. The reduction programme is gauged by the blood tests when we cannot find any other cause, such as infection. ${ }^{15}$ In these conditions, we identified the HLA DRB ${ }^{\star} 04$ antigens as a possible marker for corticosteroid resistance (the frequency of this allele was $88.88 \%$ in GCA patients with corticosteroid resistance versus $37.5 \%$ in those without corticosteroid resistance: uncorrected $\chi^{2} 7.42$, p 0.0064 RR $2.371 .43<\mathrm{RR}<3.92$, $95 \mathrm{CI} \%$, Fisher's exact tailed test, p value $0.008)$. We suggest that the GCA associated HLA DR may be used as a severity factor in confirmed GCA and can identify GCA patients at highest risk for resistant disease. These patients should be carefully followed up in pro- spective studies of longer duration to confirm that HLA HLA DRB $1^{\star} 04$ antigens may be a true marker for corticosteroid ressistance. Publications describing the use of a corticosteroid and a second immunosuppressor agent in GCA are of two kinds: those that consider so called corticosteroid resistant disease in which a second agent is added after resistance is recognised, and those that attempt to demonstrate the superiority of starting treatment with two agents rather than with a corticosteroid alone. At all events, we have as yet insufficient data to demonstrate that treatment is more effective if an immunosuppressive drug is added. ${ }^{10}$ Prospective regimens including patients with GCA who are likely to develop corticosteroid resistant disease could provide more information. Our results indicate that carriers of HLA DRB $1{ }^{\star} 04$ may be potential candidates in studies evaluating complementary treatment with immunosuppressors.

In conclusion, we confirmed that GCA is strongly associated with HLA DRB $1^{\star} 04$ and we suggest that the GCA associated $\mathrm{DRB} 1^{\star} 04$ sequence may serve as a potential marker for patients who will develop significantly corticosteroid resistant disease and may benefit from alternative treatment.

1 Arnett FC. Histocompatibility typing in rheumatic diseases. Diagnostic and pronostic implications. Rheum Dis Clin North Am 1994;20:371-85.

2 Hunder GG, Lie JT, Goronzy JJ. Pathogenesis of giant cell arteritis. Arthritis Rheum 1993;36:757-61.

3 Weyand MC, Hicok KC, Hunder GG. The HLA-DRB1 locus as a genetic component in giant cell arteritis mapping of a disease-linked sequence motif to the antigen binding site of the HLA-DR molecule. J Clin Invest 1992;90:235561.

4 Behn AR, Perera T, Myles AB. Polymyalgia rheumatica and orticosteroids: how much for how long? Ann Rheum Dis 1983;42:374-8.

5 Wagner U, Kaltenhauzer S, Sauer H. HLA markers and prediction of clinical course and outcome in rheumatoid arthritis. Arthritis Rheum1997;40:341-51.

6 Weyand CM, Hunder NNH, Hicok KC. HLA-DRB1 alleles in polymyalgia rheumatica, giant cell arteritis, and rheumatoid arthritis. Arthritis Rheum 1994;37:514-20.

7 Hunder GG, Bloch DA, Michel BA. The American College of Rheumatology 1990 criteria for the classification of giant of Rheumatology 1990 criteria for the classification

8 Bignon JD, Fernandez-Vina M. XIIth International Histocompatibility Workshop. Technical handbook. In: Charron Fauchet, ed. Paris: HLA et Médecine, 1995.

9 Bodmer JG, Marsh SGE, Albert ED. Nomenclature for factors of the HLA system, 1994. Tissue Antigens 1994;44:118.

10 Wilke WS, Hoffman GS. Treatment of corticosteroidresistant giant cell arteritis. Rheum Dis Clin North Am 1995;21:59-71.

11 Cid MC, Ercilla G, Vilaseca J. Polymyalgia rheumatica: a syndrome associated with HLA-DR4 antigen. Arthritis syndrome associated with

12 Weyand CM, Goronzy JJ. Giant cell arteritis as an antigendriven disease. Rheum Dis Clin North Am 1995;21:102739

13 Cimmino MA. Genetic and environmental factors in polymyalgiar rheumatica. Ann Rheum Dis 1997;56:576-7. 4 Kyle V, Hazleman BL. Treatment of polymyalgia rheumatica and giant cell arteritis. Relation between steroid dose and steroid associated side effects. Ann Rheum Dis 1989;48:662-6.

15 Delecoeuillerie G, Joly E, Cohen De Lara A. Polymyalgia rheumatica and temporal arteritis : a retrospect of analysis of prognostic feature and different corticosteroid arteritis (eleven year survey of 210 patients). Ann Rheum Dis 1988 ; 47:733-9. 